\title{
Sensor Networks for Diffusion Fields: Detection of Sources in Space and Time
}

\author{
Ivan Dokmanić, Juri Ranieri, Amina Chebira and Martin Vetterli \\ School of Computer and Communication Sciences \\ Ecole Polytechnique Fédérale de Lausanne (EPFL) \\ Lausanne, Switzerland
}

\begin{abstract}
We consider the problem of reconstructing a diffusion field, such as temperature, from samples collected by a sensor network. Motivated by the fast decay of the eigenvalues of the diffusion equation, we approximate the field by a truncated series. We show that the approximation error decays rapidly with time. On the other hand, the information content in the field also decays with time, suggesting the need for a proper choice of the sampling strategy. We propose two algorithms for sampling and reconstruction of the field. The first one reconstructs the distribution of point sources appearing at known times using the finite rate of innovation (FRI) framework. The second algorithm addresses a more difficult problem of estimating the unknown times at which the point sources appear, in addition to their locations and magnitudes. It relies on the assumption that the sources appear at distinct times. We verify that the algorithms are capable of reconstructing the field accurately through a set of numerical experiments. Specifically, we show that the second algorithm successfully recovers an arbitrary number of sources with unknown release times, satisfying the assumption. For simplicity, we develop the 1-D theory, noting the possibility of extending the framework to more general domains.

Index Terms-Diffusion field, source localization, release time, estimation, sensor networks, sparse sampling
\end{abstract}

\section{INTRODUCTION}

Sensor networks are often deployed to sense a phenomenon that can be described by a physical model. Knowing the underlying model allows us to reduce the quantity of measurements or to improve their quality, even if in practice this approach raises many challenges. In particular, physical models are only approximate and their parameters are usually unknown. Moreover, the theory of multidimensional sampling usually assumes that the different dimensions of the signal are homogeneous and interchangeable. This is true in images and some volumetric data, but in physical fields space and time are not equivalent.

Consider a sensor network collecting samples of a field; the spatial sampling density is limited by the dimensions of the sensors and by their price, while the temporal sampling frequency is limited by the characteristics of the analog-todigital converters, by the channel capacity, and by the energy constraints. It is often easier and cheaper to increase the temporal sampling frequency or the channel capacity, than to increase the number of sensors.

In this paper, we analyze the inverse problem of reconstructing a diffusion field from samples collected by a sensor network. Inverse problems are often ill-conditioned, meaning

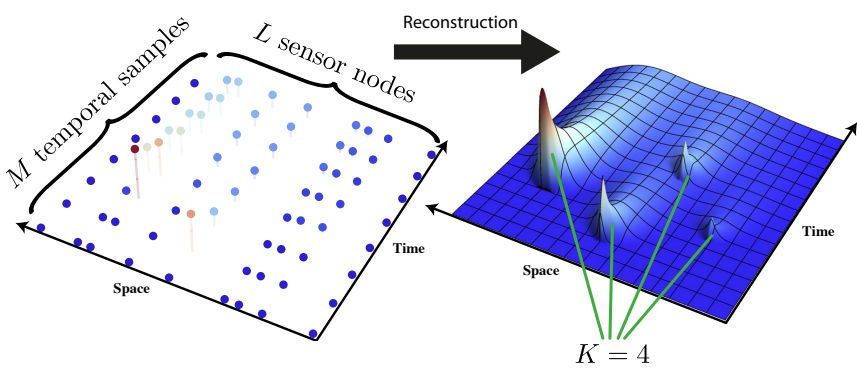

Fig. 1: Illustration of the problem setup. We show how to reconstruct the diffusion field driven by $K$ instantaneous sources from spatio-temporal samples (how to go from the left hand side to the right hand side of the figure).

that small errors in the measured data can lead to large errors in the solution. The reconstruction of diffusion fields is known to be particularly ill-conditioned [1].

Beyond the application to temperature estimation in solid bodies, we are interested in pollution detection [2], plume sources detection [3], short pulse laser applications [4], and temperature distribution estimation in a server room [5], in which the proposed techniques may be used to localize the so-called cold and hot spots, responsible for the energy inefficiency.

\section{A. Prior art}

One of the earliest works, by Fourier and Kelvin [6], studies the estimation of the initial temperature of Earth from the current temperature distribution. More recently, Al Masood et al. considered a well conditioned damped heat equation [7] and a Bessel operator [8] to estimate the initial temperature distribution in a diffusion field. Nakamura et al. [9] used transform techniques to solve the initial inverse problem in heat conduction, while Takeuchi et al. [10] proved the existence of the solution and gave a numerical method to find point sources distributed on a $2-D$ domain. In the signal processing community, one of the earliest approaches is by Nehorai et al. [11] who studied the localization of vaporemitting sources. Lu and Vetterli introduced two different approaches to the reconstruction of a sparse source distribution driving the diffusion field based on spatial super-resolution [12], and on an adaptive spatio-temporal sampling scheme 
[13]. An approach using compressed sensing techniques on a discrete grid was proposed by Ranieri et al. in [14].

\section{B. Main Contributions}

Given a set of samples in space and time, we study the reconstruction of a diffusion field driven by point sources. First, we show that the signal-to-noise ratio (SNR) of the collected samples and the effective bandwidth of the field are fast decreasing functions of time. These two figures of merit define the natural tradeoff for the sampling of diffusion sources and indicate the existence of a "sweet spot" sampling window.

We then propose a non-iterative algorithm for the estimation of an initial point source distribution and an online algorithm able to estimate the release times. The latter enables us to recover the field parameters in real-time. To ensure recovery, we require the releases to take place at distinct times. Both algorithms use overparameterization to transform a nonlinear problem into a larger linear system of equations. The source parameters are then obtained using the annihilation filter method, common in finite rate of innovation (FRI) sampling [15], [16]. For simplicity, we develop the theory in one dimension. We note that the proposed framework can be extended to more general domains.

Finally, we show through a series of numerical experiments that the proposed algorithms accurately reconstruct the field. We emphasize the experiments that verify the capability of the second algorithm to recover an arbitrary number of sources with unknown release times - a scenario not well developed in the literature. The problem setup is illustrated in Fig. 1.

\section{Paper Outline}

In Section II we present the derivation of the eigenfunction solution of the diffusion equation, which establishes the basis for the approximation used in the remainder of the paper. We also briefly analyze the approximation error. Section III and Section IV describe the proposed algorithms for the reconstruction of the diffusion field from samples. Numerical experiments are presented in Section $\mathrm{V}$ before giving the directions for future research and concluding the paper in Section VI.

\section{Diffusion EQUATION}

A one-dimensional diffusion field driven by a source distribution $s(x, t)$ satisfies the following partial differential equation (PDE),

$$
\frac{\partial f(x, t)}{\partial t}=\gamma \frac{\partial^{2} f(x, t)}{\partial x^{2}}+s(x, t) .
$$

For simplicity, we choose the units in (1) so that the diffusion coefficient is unitary, $\gamma=1$. The solution to (1) is given as a convolution

$$
f(x, t)=\int_{0}^{t} \int_{\mathbb{R}} g_{0}(x-\xi, t-\tau) s(\xi, \tau) \mathrm{d} \xi \mathrm{d} \tau,
$$

where the kernel

$$
g_{0}(x, t)=\frac{\exp \left[-x^{2} /(4 t)\right]}{2 \sqrt{\pi t}}
$$

is called the Green's function of the free-space diffusion equation [17]. We now impose a periodic boundary condition by requiring that

$$
f(x+2 k \pi, t)=f(x, t), \quad \forall k \in \mathbb{Z} .
$$

Note that having a periodic domain is not at all crucial for the proposed algorithms to be applicable. We make this choice so that the eigenvalue solution simplifies to the spatial Fourier series of the field. The Green's function changes accordingly to the following form

$$
g(x, t)=\sum_{k \in \mathbb{Z}} g_{0}(x-2 k \pi, t) .
$$

For a linear PDE, the Green's function is just the spatiotemporal impulse response corresponding to a point source appearing at $x=0$ and $t=0$.

\section{A. Eigenfunction Solution}

Consider the diffusion field $f$ on a circle of length $2 \pi$. That is, the mapping $(x, t) \mapsto f(x, t)$ is $2 \pi$-periodic in $x$. In addition, let the field be driven by a point source occurring at time $t=0$ and at location $x=x_{0}$. Namely, we have

$$
\begin{aligned}
\frac{\partial^{2} f}{\partial x^{2}}-\frac{\partial f}{\partial t} & =\delta\left(x-x_{0}\right) \delta(t), \\
f(x+2 k \pi, t) & =f(x, t), \quad \forall k \in \mathbb{Z}, t \geq 0 .
\end{aligned}
$$

We know that the solution to this equation is given by the convolution of the source distribution $s(x, t)$ (3) with the Green's function $g(x, t)$. For our purposes, a more convenient expression for the Green's function is obtained by computing the eigenfunction solution to (6).

Assume that $f(x, t)=\chi(x) \mathrm{e}^{-\omega^{2} t}$, where $\chi$ does not depend on time. This gives

$$
\mathrm{e}^{-\omega^{2} t} \frac{\partial^{2} \chi}{\partial x^{2}}+\omega^{2} \mathrm{e}^{-\omega^{2} t} \chi(x)=0 .
$$

We require (7) to hold for every $t$ so this is equivalent to

$$
\frac{\partial^{2} \chi}{\partial x^{2}}=-\omega^{2} \chi(x)
$$

Solutions to (8) are $\sin (\omega x), \cos (\omega x)$ and $\mathrm{e}^{\mathrm{i} \omega x}$. The periodic boundary condition forces $\omega$ 's into the form $\omega_{n}=n$, so the homogeneous solution to (6) corresponding to $\omega_{n}$ is

$$
f_{n}(x, t)=\mathrm{e}^{\mathrm{i} \omega_{n} x} \mathrm{e}^{-\omega_{n}^{2} t}=\mathrm{e}^{\mathrm{i} n x} \mathrm{e}^{-n^{2} t} .
$$

The general homogeneous solution is just a linear combination of $\left\{f_{n}\right\}_{n \in \mathbb{Z}}$,

$$
f(x, t)=\sum_{n \in \mathbb{Z}} c_{n} \mathrm{e}^{\mathrm{i} n x} \mathrm{e}^{-n^{2} t},
$$

where the coefficients $c_{n}$ are computed from the given initial value of $f$.

A Dirac delta source at $t=0$ can be cast as an initial value by requiring that $f(x, t) \rightarrow \delta\left(x-x_{0}\right)$ as $t \rightarrow 0$, or equivalently,

$$
\int_{A} f(x, t) \mathrm{d} x=1, \quad \text { as } t \rightarrow 0
$$


for every open set $A \subset \mathbb{S}^{1}$ containing $x_{0}$, where $\mathbb{S}^{1}$ is the unit circle. Combining (10) with this initial condition yields the coefficients $c_{n}$ as the coefficients of a basis expansion of a Dirac in the orthogonal basis of eigenfunctions $\left\{\mathrm{e}^{\mathrm{i} n x}\right\}_{n \in \mathbb{Z}}$. The general solution with the initial value (11) can therefore be written as

$$
f(x, t)=\frac{1}{2 \pi} \sum_{n \in \mathbb{Z}} \mathrm{e}^{-\mathrm{i} n x_{0}} \mathrm{e}^{\mathrm{i} n x} \mathrm{e}^{-n^{2} t}=\frac{1}{2 \pi} \sum_{n \in \mathbb{Z}} \mathrm{e}^{\mathrm{i} n\left(x-x_{0}\right)-n^{2} t} .
$$

This is an alternative form of the Green's function (5) for the initial boundary value problem (6).

The elements of the series in (12) decay rapidly to zero due to the $\mathrm{e}^{-n^{2} t}$ term, so we can approximate the sum by truncating the series. To do so, we keep $2 N+1$ elements centered around $n=0$,

$$
f(x, t) \approx \frac{1}{2 \pi} \sum_{n=-N}^{N} \mathrm{e}^{\mathrm{i} n\left(x-x_{0}\right)-n^{2} t}
$$

The choice of $N$ is discussed in Section II-B. Note that this means that the field is approximately bandlimited in space, for times away from 0 .

\section{B. Tradeoffs in Diffusion Sampling}

The proposed diffusion field sampling involves three quantities: spatial sampling frequency, temporal sampling frequency, and the cutoff index $N$. If we sample too late after the source had appeared, the SNR is too low to make any reasonable inference from these samples. On the other hand, if we sample too close to the source (in time and space), the bandwidth is large. Therefore, for a fixed $N$, the truncated approximation may be inaccurate, leading to the failure of the reconstruction algorithm. This shows that we should choose $N$ according to the desired spatial and temporal sampling frequency. We formalize this reasoning in the following proposition.

Proposition 1. Let $f(x, t)$ be a diffusion field on a unit circle, driven by a point source of unit intensity appearing at $(t, x)=\left(0, x_{0}\right)$. Furthermore, let $f^{(N)}(x, t)$ be the $(2 N+1)-$ term approximation of $f(x, t)$. Then the following bound on the relative approximation error holds,

$\left|\frac{f(x, t)-f^{(N)}(x, t)}{f(x, t)}\right| \leq 4 \sqrt{\pi t} \exp \left[\frac{\left\|x-x_{0}\right\|^{2}}{4 t}\right] \sum_{n>N} \mathrm{e}^{-n^{2} t}$.

Furthermore, $f(x, t) \rightarrow 1 /(2 \pi)$ as $t \rightarrow \infty$.

The Proposition 1 shows that the approximation error decays rapidly in time, and that the SNR goes to zero with time. We omit the proof here since it is purely technical and does not add new insight.

The bound on the approximation error (14) is drawn in Fig. 2. The same figure also depicts the corresponding field as a function of time, to show that the error decays much faster than the field.
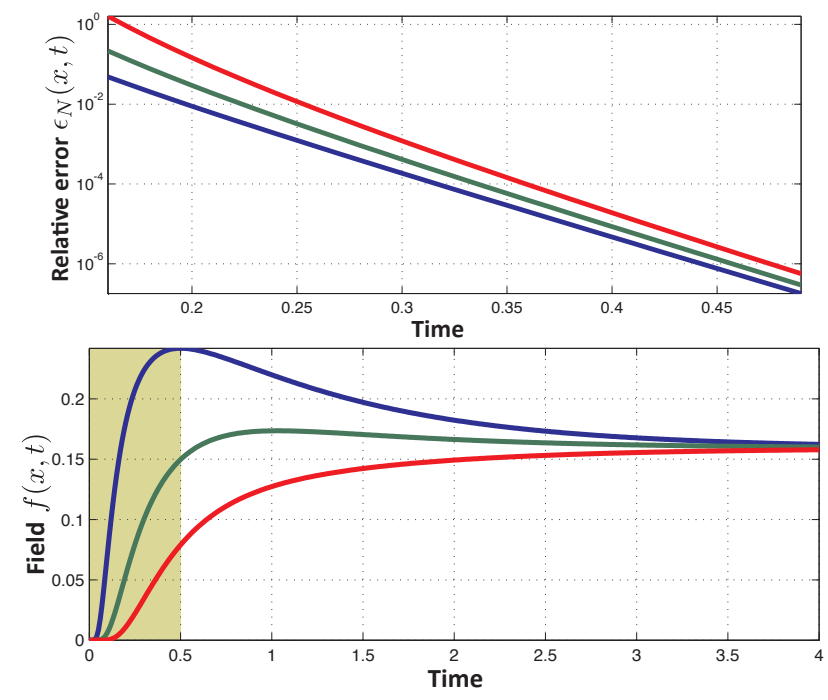

Fig. 2: Bound on the relative approximation error as a function of time for three sensor locations (a), and the field value as a function of time at the same locations (b). The approximation error is shown for the truncation order $N=5$. Note the difference in the temporal axis limits, indicating the "sweet spot" sampling window where the error is negligible, and the field samples still carry information (since the error keeps decaying rapidly, we show it only up to $t=0.5 \mathrm{~s}$ ).

\section{Solving the Initial Source Problem}

In this section, we give a step-by-step derivation of the algorithm for reconstructing the initial distribution of diffusion point sources. These steps consider various scenarios of increasing complexity.

\section{A. Step 1: One unit source, one sample}

If we have a sensor at location $\xi$ observing the field at time $\tau$, then we have access to

$$
f(\xi, \tau) \approx \frac{1}{2 \pi} \sum_{n=-N}^{N} \mathrm{e}^{\mathrm{i} n\left(\xi-x_{0}\right)-n^{2} \tau}
$$

Let $\alpha_{n} \stackrel{\text { def }}{=} \frac{1}{2 \pi} \mathrm{e}^{\mathrm{i} n \xi-n^{2} \tau}, z=\mathrm{e}^{-\mathrm{i} x_{0}}$, and $\phi=f(\xi, \tau)$. Assuming that $\alpha_{n}$ is known by design, we obtain an equation in $z$ of the form

$$
\sum_{n=-N}^{N} \alpha_{n} z^{n} \approx \phi
$$

After multiplying by $z^{N}$ this becomes a polynomial equation. It is not difficult to compute the roots of (16) and find the source location. Due to symmetry, we obtain two feasible locations. We only use this very simple case to illustrate the principle and to establish the notation, without discussing it further. 


\section{B. Step 2: One unit source, multiple samples}

Consider now the set of measurements $\left\{\phi_{m}\right\}_{m=1}^{M}$, taken at different times and different locations. Arrange the measurements in a vector $\phi$ such that $\phi[m]=\phi_{m}$. Let also $\alpha_{m n}=\alpha_{m n}\left(\xi_{m}, \tau_{m}\right)$ be the coefficient multiplying $z^{n}$ in the summation (16), corresponding to the $m$ th measurement, and define the matrix $\boldsymbol{A}$ by $\boldsymbol{A}[m, n]=\alpha_{m n}$. Finally, let $\boldsymbol{z}$ denote the vector $\left[z^{-N}, z^{-(N-1)}, \ldots, z^{N-1}, z^{N}\right]^{T}$. Then, if we treat the powers of $z$ as independent linear unknowns, we have the following linear system of equations

$$
A z \approx \phi
$$

Provided that the measurement setup is $\operatorname{such}$ that $\operatorname{rank}(\boldsymbol{A})=$ $2 N+1$, we can find $z \approx \mathrm{e}^{-\mathrm{i} x_{0}}$ by solving the linear system (17).

Note that here it is necessary to further justify the meaning of $\approx$, by examining the conditioning of the matrix $\boldsymbol{A}$. This computation is beyond the scope of this paper.

\section{Step 3: Multiple sources, multiple samples}

Let the $K$ diffusion sources with magnitudes $\left\{s_{k}\right\}_{k=1}^{K}$ be located at $\left\{x_{k}\right\}_{k=1}^{K}$. Then the measurement (16) can be rewritten as follows

$$
\sum_{n=-N}^{N} \alpha_{m n}\left(s_{1} z_{1}^{n}+s_{2} z_{2}^{n}+\cdots s_{K} z_{K}^{n}\right) \approx \phi_{m}, 1 \leq m \leq M .
$$

We can again write (18) in a matrix form $\boldsymbol{A} \boldsymbol{z}=\boldsymbol{\phi}$, where we have redefined $\boldsymbol{z}$ as $\boldsymbol{z}[n] \stackrel{\text { def }}{=} s_{1} z_{1}^{n}+\cdots+s_{K} z_{K}^{n}$. Repeating the reasoning from the previous step, if we treat each $\boldsymbol{z}[n]$ as being independent, we can find $z$ by solving a linear system, provided that $\operatorname{rank}(\boldsymbol{A})=2 N+1$. After computing $\boldsymbol{z}$, we have the following nonlinear system of equations,

$$
\left\{\begin{array}{lll}
s_{1} z_{1}^{-N} & +\cdots+s_{K} z_{K}^{-N} & \approx \boldsymbol{z}[-N] \\
s_{1} z_{1}^{-(N-1)} & +\cdots+s_{K} z_{K}^{-(N-1)} & \approx \boldsymbol{z}[-(N-1)] \\
\vdots & \\
s_{1} z_{1}^{N} & +\cdots+s_{K} z_{K}^{N} & \approx \boldsymbol{z}[N]
\end{array}\right.
$$

The signal $z$ is a sum of $K$ complex exponentials. To find the frequencies of the individual exponentials, we use the annihilation filter method [15]. Intensities $\left\{s_{k}\right\}_{k=1}^{K}$ are then found by solving a linear system.

From here on, we consider the following sampling scenario: consider $K$ sources at locations $\left\{x_{k}\right\}_{k=1}^{K}$ with intensities $\left\{s_{k}\right\}_{k=1}^{K}$. Assume that we have $L$ sensors at locations $\left\{\xi_{l}\right\}_{l=1}^{L}$, each of which takes $M$ measurements at times $\left\{\tau_{m}\right\}_{m=1}^{M}$. This results in a nonuniform but rectangular spatio-temporal sampling lattice. Note that the proposed solution does not require any regularity of the sampling lattice. The recovery of the initial source distribution is summarized in Algorithm 1.

We conclude this part by noting that the sampling process can be described in a compact matrix form. Let $\boldsymbol{\Phi}$ be an $M \times L$

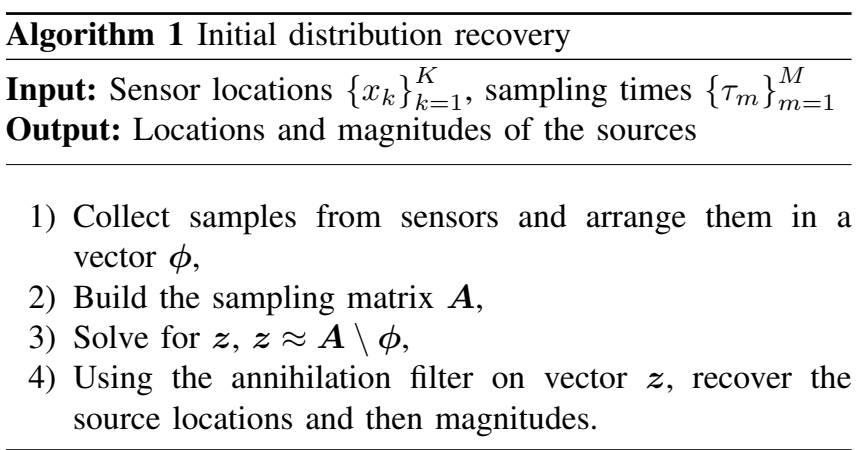

matrix such that $\boldsymbol{\Phi}[m, l]=f\left(\xi_{l}, \tau_{m}\right), \boldsymbol{T}$ an $M \times(2 N+1)$ matrix such that $\boldsymbol{T}[m, n]=\mathrm{e}^{-n^{2} \tau_{m}}, \boldsymbol{Z}$ a diagonal $(2 N+$ $1) \times(2 N+1)$ matrix such that $\boldsymbol{Z}[n, n]=\sum_{k} s_{k} z_{k}^{n}$, and $\boldsymbol{\Xi}$ a $(2 N+1) \times L$ matrix such that $\boldsymbol{\Xi}[n, l]=\mathrm{e}^{\mathrm{i} i \xi_{l}}$. Then we have the following,

$$
\Phi \approx T Z \Xi
$$

where the matrix $\boldsymbol{T}$ depends only on the temporal sampling pattern, the matrix $\boldsymbol{\Xi}$ depends only on the spatial sensor network configuration, and the matrix $Z$ depends on the source locations. For clarity, we give an expanded form of (20) in (21).

\section{SPATIO-TEMPoral RECONSTRUCtion OF FiELdS WITH BOUNDED RELEASE RATE}

A challenging issue in spatio-temporal diffusion field reconstruction is the reconstruction of the unknown release times. We propose an algorithm to reconstruct both the unknown release times and the unknown source locations. The proposed algorithm is an online algorithm. That is, it assumes that the deployed sensor network uninterruptedly samples the field with a given sampling frequency. For the algorithm to operate correctly we must constrain the allowed temporal release patterns. This is summarized in the following definition.

Definition 1. We say that a diffusion field driven by point sources appearing at times $\left\{t_{k}\right\}_{k=1}^{\infty}$ has a bounded release rate if $D=\inf _{i \neq j}\left|t_{i}-t_{j}\right|>0$. We will call $1 / D$ the maximum release rate.

The proposed algorithm is applicable to diffusion fields with a bounded release rate. We argue that essentially all interesting fields have a bounded release rate. The temporal sampling frequency must be chosen according to the maximum release rate of the field. For such fields, we show that the algorithm is capable of estimating parameters for an arbitrary number of sources.

Since we do not know where and when the releases take place, and since the quality of the information available from the field decays rapidly with time (see Proposition 1), we must ensure that the spatial sampling is sufficiently dense. We leave the precise theoretical analysis to future work.

We justify this algorithm by causality and fast decay of the field. Causality means that there is absolutely no information in the field about sources that are yet to appear. Each source 
introduces the need to sample it, but only after it had appeared. Therefore, we argue that any robust reconstruction scheme must sample continuously.

1) Estimating the release time for one source: A field generated by a point source appearing at $\left(t_{0}, x_{0}\right)$ with magnitude $s_{0}$ is given as

$$
f(x, t) \approx \frac{s_{0}}{2 \pi} \sum_{n=-N}^{N} \mathrm{e}^{\mathrm{i} n\left(x-x_{0}\right)-n^{2}\left(t-t_{0}\right)},
$$

where we assume that the choice of $N$ is such that the approximation error is negligible. As before, we assume we have $L$ sensors at locations $\left\{\xi_{l}\right\}_{l=1}^{L}$, each of which takes $M$ measurements at times $\left\{\tau_{m}\right\}_{m=1}^{M}$. Let $\beta_{m n} \stackrel{\text { def }}{=} \frac{1}{2 \pi} \mathrm{e}^{-n^{2} \tau_{m}+\mathrm{i} n \xi_{m}}$ and $y_{n} \stackrel{\text { def }}{=} s_{0} \cdot \mathrm{e}^{-\mathrm{i} n x_{0}+n^{2} t_{0}}$. Arranging the measurements in a vector $\phi$ of length $L M$, we obtain the following system of equations,

$$
\sum_{n=-N}^{N} \beta_{m n} y_{n} \approx \phi_{m}, \quad m \in\{1, \ldots, L M\} .
$$

We can again cast this system of nonlinear equations as a linear system by treating the $y$ 's as independent linear unknowns,

$$
B y \approx \phi
$$

The estimation of the parameters of one source is summarized in the following proposition.

Proposition 2. Consider the diffusion field $f(x, t)$ driven by a point source of intensity $s_{0}$, appearing at $\left(t_{0}, x_{0}\right)$. Let the sampling setup be such that all the samples are accurately represented by an N-term truncation. Then the source parameters are given as follows,

$$
\begin{aligned}
s_{0} & \approx \boldsymbol{y}[0], \\
t_{0} & \approx \log \left|\boldsymbol{y}[-1] / s_{0}\right|, \\
x_{0} & \approx \arg \boldsymbol{y}[-1] .
\end{aligned}
$$

Proof: Straightforward from the definition of $y_{n}$.

One might be tempted to simply take $N=1$ and have a very small system, requiring a small number of measurements. This is not feasible since the assumed field model would be inaccurate. We note again that it is necessary to precise the meaning of $\approx$ by taking into account the conditioning of $\boldsymbol{B}$.

A. Online estimation of parameters for an arbitrary number of sources

We now give a list of observations which, together with Proposition 2, enable us to design an efficient spatio-temporal reconstruction algorithm that can estimate release times, locations and intensities:

1) After we detect one source, we can cancel it from subsequent measurements, by simply subtracting the estimated value of the field generated by that source.

2) For the estimation algorithm in Proposition 2 to be correct, the sensor network must collect $N_{\text {samp }}$ samples, where $N_{\text {samp }}$ depends on the network's configuration, and we assume that it is known. If we collect a sample too close in time to the release (for an exact idea see Fig. 2), the truncated model will be inaccurate. We avoid this problem by discarding the first $N_{\text {guard }}$ samples before running the estimation.

3) If we use absolute time in the estimation algorithm, the coefficients $\beta$ in (23) become too small for the machine precision due to the $\mathrm{e}^{-n^{2} \tau}$ term. This is avoided if we use local time by rewriting (22) as follows,

$$
f(x, t) \approx \frac{s_{0}}{2 \pi} \sum_{n=-N}^{N} \mathrm{e}^{\mathrm{i} n\left(x-x_{0}\right)-n^{2}\left(t-t_{\text {off }}-\left(t_{0}-t_{\text {off }}\right)\right)},
$$

where $t_{\text {off }}$ is arbitrary, and we choose it to ensure the numerical stability of the estimation. Note that $t_{\text {off }}$ changes for each sample.

The complete online estimation process is presented in Algorithm 2.

\begin{tabular}{l}
\hline Algorithm 2 Online reconstruction of diffusion field \\
Input: $N_{\text {samp }}, N_{\text {guard }}$, maximum release rate $1 / D$, detection \\
threshold
\end{tabular}

Output: Release times $\boldsymbol{t}$, locations $\boldsymbol{x}$ and magnitudes $s$

1) Compute the sampling period as $\Delta=\frac{D}{N_{\text {samp }}+N_{\text {guard }}}$,

2) Initialize circular buffers of depth $N_{\text {samp }}+N_{\text {guard }}$ to store magnitudes $s$, locations $\boldsymbol{x}$ and times $\boldsymbol{t}$ Of the detected sources,

3) Repeat:

(i) $j \leftarrow j+1$

(ii) Collect samples at $j \Delta$,

(iii) For $k \in 1: \#$ (detected sources)

- Subtract $\boldsymbol{s}[k] g(t-\boldsymbol{t}[k], x-\boldsymbol{x}[k])$ from the collected samples,

(iv) Buffer the samples for $t=j \Delta$,

(v) If min(collected samples) $<$ detection threshold,

- Goto (i),

otherwise

- Discard the oldest $N_{\text {guard }}$ samples,

- Compute $\boldsymbol{y}$ according to (24),

- $s_{0} \leftarrow \boldsymbol{y}[0], t_{0} \leftarrow \log \left|\boldsymbol{y}[-1] / s_{0}\right|, x_{0} \leftarrow$ arg $\boldsymbol{y}[-1]$,

- Add $s_{0}, t_{0}$ and $x_{0}$ to $s, \boldsymbol{x}$ and $\boldsymbol{t}$, and increment the number of detected sources,

- Clear sample buffer.

\section{NUMERICAL EXPERIMENTS}

We have validated both algorithms on a number of numerical simulations, which we present in Fig. 3 and Fig. 4. In Fig. 3 we show the reconstructed source locations and the field profile for a field generated by four randomly located sources. The sensing network comprises 12 sensors, each taking four samples of the field, and the source locations are estimated using Algorithm 1. 


$$
\begin{aligned}
& {\left[\begin{array}{ccc}
f\left(\xi_{1}, \tau_{1}\right) & \cdots & f\left(\xi_{L}, \tau_{1}\right) \\
\vdots & \ddots & \vdots \\
f\left(\xi_{1}, \tau_{M}\right) & \cdots & f\left(\xi_{L}, \tau_{M}\right)
\end{array}\right]} \\
& \approx \frac{1}{2 \pi}\left[\begin{array}{ccc}
\mathrm{e}^{-(-N)^{2} \tau_{1}} & \cdots & \mathrm{e}^{-N^{2} \tau_{1}} \\
\vdots & \ddots & \vdots \\
\mathrm{e}^{-(-N)^{2} \tau_{M}} & \cdots & \mathrm{e}^{-N^{2} \tau_{M}}
\end{array}\right]\left[\begin{array}{cccc}
\sum_{k} s_{k} z_{k}^{-N} & 0 & \cdots & 0 \\
0 & \sum_{k} s_{k} z_{k}^{-(N-1)} & \cdots & 0 \\
0 & 0 & \ddots & 0 \\
0 & 0 & 0 & \sum_{k} s_{k} z_{k}^{N}
\end{array}\right]\left[\begin{array}{ccc}
\mathrm{e}^{\mathrm{i}(-N) \xi_{1}} & \cdots & \mathrm{e}^{\mathrm{i}(-N) \xi_{L}} \\
\vdots & \ddots & \vdots \\
\mathrm{e}^{\mathrm{i} N \xi_{1}} & \cdots & \mathrm{e}^{\mathrm{i} N \xi_{L}}
\end{array}\right]
\end{aligned}
$$

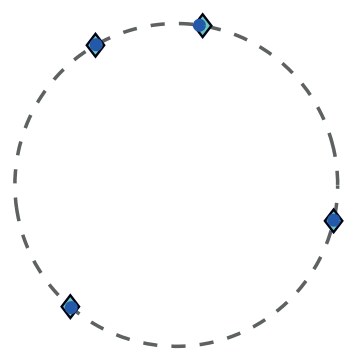

(a)

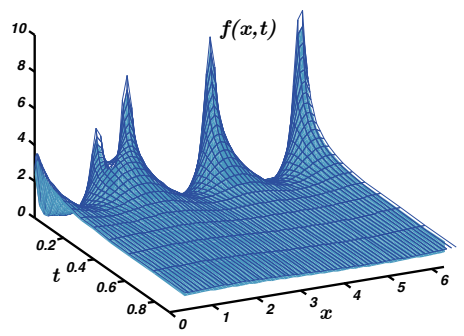

(d)

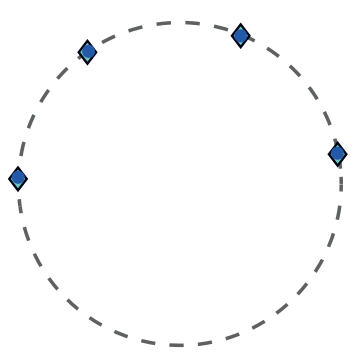

(b)

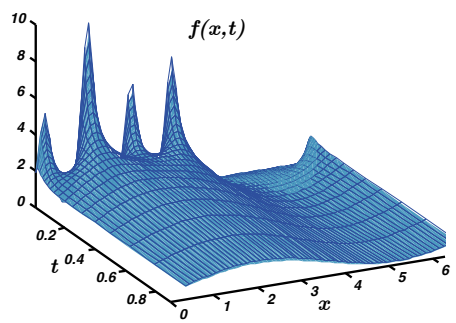

(e)

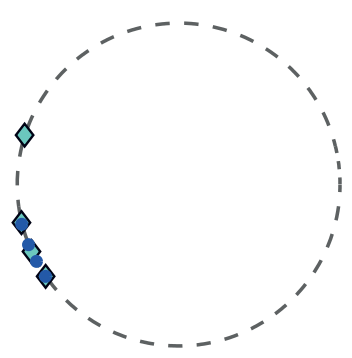

(c)

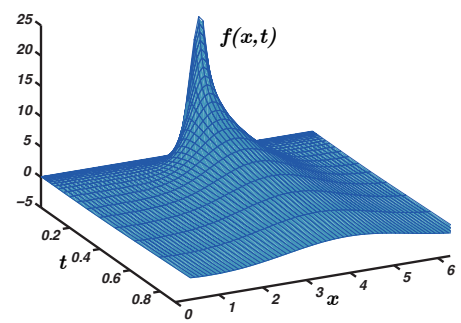

(f)

Fig. 3: Reconstruction of the source configuration in an initial value problem. The field is generated by 4 randomly located sources. True source positions in (a), (b) and (c) are marked by blue dots, while the estimated locations are denoted with a diamond. The reconstructed field is shown in (d), (e) and (f) as a solid surface, where the unit circle is mapped onto an interval for visualization. The mesh represents the true field. Even if in the case (c) some source locations are inaccurately estimated, the field estimation is accurate.

Diamonds denote the reconstructed sources, while blue dots denote the actual source locations. Field is shown as a solid blue surface, superposed with a mesh that shows the true field.

We conclude from Fig. 3a) and Fig. 3b) that the source locations are accurately reconstructed. From the field surface plots, Fig. 3d) and Fig. 3e), we further conclude that the magnitudes are estimated correctly.

We deliberately show a degenerate case in Fig. 3c), and Fig. 3f) to conclude that even when some of the sources are not localized correctly, the error in the field reconstruction is small. Intuitively, the reason for inaccurate localization is that the sources are all clustered together, yielding a unimodal field as shown in Fig. 3f).

Fig. 4 shows the reconstruction of two different diffusion fields driven by sources randomly appearing in both space and time. The diffusion fields have bounded release rates, as established in Definition 1, and the sources are retrieved using
Algorithm 2. In Fig. 4a) and Fig. 4d) we show the release times, locations and magnitudes of the estimated sources (blue), compared to the parameters of the true sources (red). Spatial and temporal projections of the field are shown in Fig. 4b) and Fig. 4e), with the sampling instants denoted by red crosses. For reasons of visualization, we omit the temporal sampling instants in Fig. 4e).

The recovered fields are given as surface plots in Fig. 4c) and Fig. 4f).

\section{CONCLUSION}

We studied the reconstruction of diffusion fields from samples collected by a sensor network. Our contributions are twofold. First, we introduce an approximate model of a diffusion field based on the fast decay of the eigenvalues of a diffusion equation. We analyze the approximation error, and assert that there is a "sweet spot" temporal sampling window. 


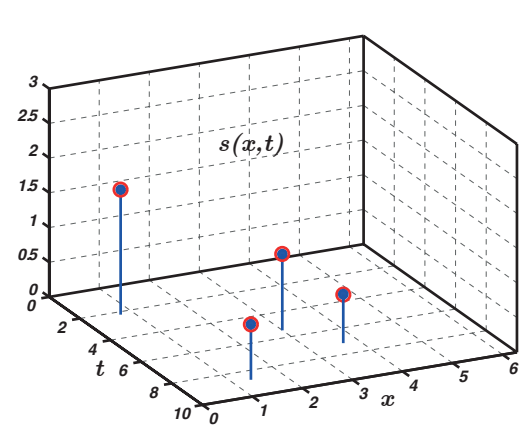

(a)

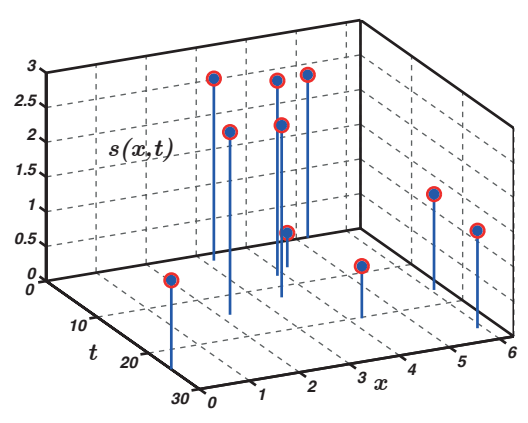

(d)
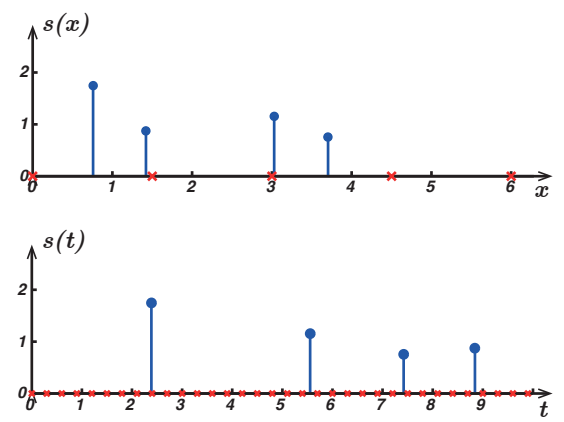

(b)
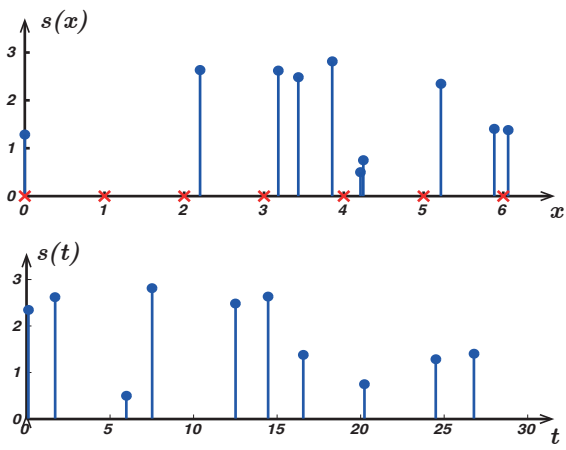

(e)

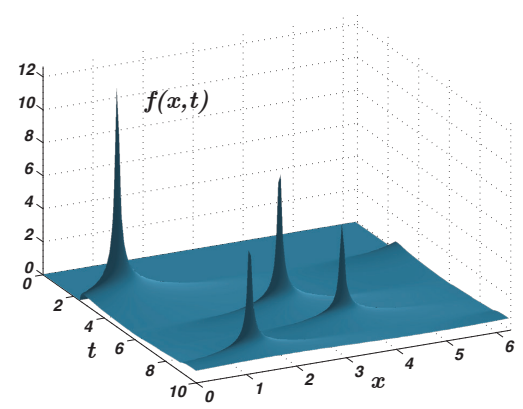

(c)

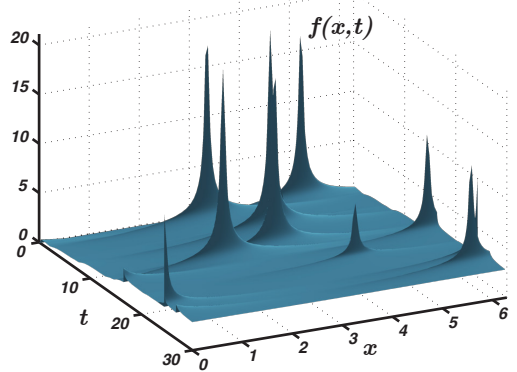

(f)

Fig. 4: Reconstruction of release times, locations and magnitudes with Algorithm 2. Reconstructed sources are shown in (a) and (d) in blue, with true sources shown as red circles. The corresponding temporal and spatial projections are given in (b) and (e). Red crosses show the sampling instants in space and time, and the reconstructed fields are shown in (c) and (f).

Second, we proposed two algorithms for field estimation. Algorithm 1 is designed for the recovery of locations and magnitudes of point sources appearing at a known time. Algorithm 2 addresses a more challenging problem of estimating the distribution of point sources with unknown release times, locations and magnitudes. We propose a solution relying on the assumption that the sources appear at distinct times. If the field satisfies this assumption, Algorithm 2 is capable of estimating the parameters of an arbitrary number of sources. We substantiate the theoretical developments through simulations, and observe accurate reconstruction of the field with both algorithms.

Ongoing work includes extensions to more general domains, a detailed theoretical analysis concerning the reconstruction performance, and a study of optimal sampling strategies.

\section{REFERENCES}

[1] C. Hansen, Rank-deficient and discrete ill-posed problems: numerical aspects of linear inversion. Soc. for Industrial and Applied Math., 1987.

[2] A. El Badia and T. Ha-Duong, "An inverse problem in heat equation and application to pollution problem," Inverse and ill-Posed Problems, 2002.

[3] J. M. Stockie, "The mathematics of atmospheric dispersion modeling," SIAM Review, 2011.

[4] A. Vedavarz, K. Mitra, and S. Kumar, "Hyperbolic temperature profiles for laser surface interactions," J. of Applied Physics, 1994.
[5] K. Chen, C. E. Bash, D. M. Auslander, and C. D. Patel, "Local temperature control in data center cooling: Part II, Statistical Analysis," Tech. Rep., 2006.

[6] H. S. Carslaw and J. C. Jaeger, Conduction of heat in solids. Oxford University Press, USA, 1986.

[7] K. Masood and F. D. Zaman, "Investigation of the Initial Inverse Problem in the Heat Equation," J. of Heat Tran., 2004.

[8] K. Masood, S. Messaoudi, and F. D. Zaman, "Initial inverse problem in heat equation with Bessel operator," Int. J. Heat Mass Tran., 2002.

[9] G. Nakamura, S. Saitoh, and A. Syarif, "Representations of initial heat distributions by means of their heat distributions as functions of time," Inverse Probl., 1999.

[10] L. Ling, M. Yamamoto, Y. C. Hon, and T. T, "Identification of source locations in two-dimensional heat equations," Inverse Probl., 2006.

[11] A. Nehorai, B. Porat, and E. Paldi, "Detection and localization of vaporemitting sources," IEEE Transactions on Signal Processing, 1995.

[12] Y. M. Lu and M. Vetterli, "Spatial super-resolution of a diffusion field by temporal oversampling in sensor networks," in Proc. IEEE Int. Conf. Acoust., Speech, and Signal Proc., 2009.

[13] — - "Distributed spatio-temporal sampling of diffusion fields from sparse instantaneous sources," in Proc. 3rd Int. Workshop on Comp Adv. in Multi-Sensor Adaptive Proc., 2009.

[14] J. Ranieri, A. Chebira, Y. Lu, and M. Vetterli, "Sampling and reconstructing diffusion fields with localized sources," in Proc. IEEE Int. Conf. Acoust., Speech, and Signal Proc., 2011.

[15] M. Vetterli, P. Marziliano, and T. Blu, "Sampling signals with finite rate of innovation," IEEE Transactions on Signal Processing, 2002.

[16] T. Blu, P. Dragotti, M. Vetterli, P. Marziliano, and L. Coulot, "Sparse Sampling of Signal Innovations," IEEE Signal Proc. Mag., 2008.

[17] D. Duffy, Green's functions with applications. Chapman and Hall/CRC, 2001. 